\title{
Evaluation of central nervous system in patients with glycogen storage disease type $1 \mathrm{a}$
}

\author{
Yusuf Aydemir ${ }^{1}$, Figen Gürakan ${ }^{1}$, İnci Nur Saltık Temizel ${ }^{1}$, Hülya Demir ${ }^{1}$, Kader Karlı Oğuz ${ }^{2}$, \\ Dilek Yalnızoğlư ${ }^{3}$, Meral Topçu ${ }^{3}$, Hasan Özen ${ }^{1}$, Aysel Yüce ${ }^{1}$ \\ Divisions of ${ }^{1}$ Pediatric Gastroenterology and Hepatology, and ${ }^{3}$ Pediatric Neurology, Department of Pediatrics and ${ }^{2}$ Department \\ of Radiology, Hacettepe University Faculty of Medicine, Ankara, Turkey. \\ E-mail: dryusufaydemir@yahoo.com \\ Received: 28 February 2016, Revised: 13 April 2016, Accepted: 28 April 2016
}

SUMMARY: Aydemir Y, Gürakan F, Saltık-Temizel İN, Demir H, Karlı-Oğuz K, Yalnızoğlu D, Topçu M, Özen H, Yüce A. Evaluation of central nervous system in patients with glycogen storage disease type 1a. Turk J Pediatr 2016; 58: 12-18.

We aimed to evaluate structure and functions of central nervous system (CNS) in children with glycogen storage disease (GSD) type 1a.

Neurological examination, psychometric tests, electroencephalography (EEG), magnetic resonance imaging (MRI), visual evoked potentials (VEP) and brainstem auditory evoked potentials (BAEP) were performed. The results were compared between patients with good and poor metabolic control and healthy children.

Twenty-three patients with GSD type 1a were studied. Twelve patients were in poor metabolic control group and 11 patients in good metabolic control group. Five patients had intellectual disability, 10 had EEG abnormalities, seven had abnormal VEP and two had abnormal BAEP results. MRI was abnormal in five patients. There was significant correlation between the number of hypoglycemic attacks and MRI abnormalities.

Central nervous system may be affected in GSD type 1a even in patients with normal neurologic examination. Accumulation of abnormal results in patients with poor metabolic control supports the importance of metabolic control in GSD type 1a.

Key words: glycogen storage disease type 1a, central nervous system, hypoglycemia, intellectual disability.

Glycogen storage diseases (GSD) are rare hereditary disorders affecting glycogen metabolism. Because of the defect of enzymes responsible for the synthesis or degradation of glycogen, abnormal accumulation of glycogen takes place in different organs; primarily in liver and muscles. There are over 12 types of GSD based on different enzyme deficiencies ${ }^{1}$.

Glycogen storage disease type 1 is the first identified metabolic disorder caused by an enzyme deficiency ${ }^{2}$. Glucose 6-phosphatase (G6Pase) enzyme catalyzes the terminal reaction of glycogenolysis and gluconeogenesis in hepatocytes, enterocytes and renal epithelial cells ${ }^{3}$. Deficient catalytic activity of the G6Pase system leads to GSD type 1a, which constitutes more than $80 \%$ of patients with GSD type 1 . Its incidence is estimated to be around $1 / 100,000$ births. GSD type 1a is characterized by impaired production of glucose from glycogenolysis and gluconeogenesis resulting in severe fasting hypoglycemia and increased production of lactic acid, triglyceride, and uric acid ${ }^{4}$.

Patients may present shortly after birth with hepatomegaly, hypoglycemia, tremor, irritability, apnea, seizure and lactic acidosis. They may also present later with doll like face, protuberant abdomen, lethargy, seizures and growth retardation. Anemia, bleeding tendency, diarrhea, and late onset puberty are other common findings. Patients especially with poor metabolic control have long-term complications such as short stature, developmental delay, seizures, intellectual disability, hepatic adenoma, renal 
dysfunction, gout and osteoporosis 5 . Frequent meals, continuous nocturnal drip-feeding and the administration of uncooked cornstarch are the treatment modalities commonly used either alone or in combination ${ }^{6}$. The aim of dietary treatment is to maintain normoglycemia and to prevent long-term complications of hypoglycemia. If hypoglycemia can be prevented, the biochemical and clinical abnormalities in most patients will improve ${ }^{7}$. However, in older patients, various complications may still develop. Although recurrent hypoglycemia occurs in disease course, severe central nervous system damage does not occur as severely as expected $^{8}$. We aim to evaluate structure and function of central nervous system in a group of children with GSD type 1a on long-term follow up.

\section{Material and Methods}

This study was conducted at Hacettepe University Children's Hospital, Department of Pediatric Gastroenterology and Hepatology. Twenty-three patients with GSD type 1a were enrolled. All the patients were diagnosed previously by histopathological findings, glycogen amount, and G6Pase level in liver tissue according to criteria described by Narisawa et al. ${ }^{9}$ Patients with history of prematurity, head trauma or any acute/chronic disease other than GSD type 1a

Table I. Clinical Features, Psychometric Tests, Electrophysiological and Radiological Findings in Patients with GSD Type 1a

\begin{tabular}{|c|c|c|c|c|c|c|c|c|c|c|}
\hline $\begin{array}{c}\text { Patient } \\
\text { no }\end{array}$ & Age & $\begin{array}{l}\text { Number of } \\
\text { hypoglycemic } \\
\text { attacks }\end{array}$ & Seizures & $\begin{array}{c}\text { Dietary } \\
\text { compliance }\end{array}$ & $\begin{array}{c}\text { Metabolic } \\
\text { control }\end{array}$ & $\begin{array}{l}\text { VEP } \\
\text { P100 } \\
\text { latency }\end{array}$ & $\begin{array}{c}\text { BAEP } \\
\text { I-V } \\
\text { interpeak } \\
\text { latency }\end{array}$ & EEG & MRI & $\begin{array}{c}\text { Psychometric } \\
\text { test }\end{array}$ \\
\hline 1 & 12.5 & 6 & 0 & $\mathrm{H}$ & $\mathrm{P}$ & $\mathrm{N}$ & $\mathrm{N}$ & A & $\mathrm{N}$ & $\mathrm{LAv}$ \\
\hline 2 & 10 & 3 & 1 & $\mathrm{~L}$ & G & $\mathrm{N}$ & $\mathrm{N}$ & A & $\mathrm{N}$ & $\mathrm{LAv}$ \\
\hline 3 & 3.3 & 20 & 5 & $\mathrm{~L}$ & $\mathrm{P}$ & A & $\mathrm{N}$ & - & $\mathrm{N}$ & - \\
\hline 4 & 11.5 & 4 & 0 & $\mathrm{H}$ & G & $\mathrm{N}$ & $\mathrm{N}$ & $\mathrm{N}$ & - & $\mathrm{Av}$ \\
\hline 5 & 6.5 & 2 & 0 & $\mathrm{H}$ & G & $\mathrm{N}$ & - & A & $\mathrm{N}$ & $\mathrm{Av}$ \\
\hline 6 & 14 & 2 & 0 & $\mathrm{H}$ & G & A & $\mathrm{N}$ & $\mathrm{N}$ & $\mathrm{N}$ & - \\
\hline 7 & 10.5 & 3 & 1 & $\mathrm{~L}$ & G & A & $\mathrm{N}$ & $\mathrm{N}$ & $\mathrm{N}$ & - \\
\hline 8 & 10.5 & 4 & 0 & $\mathrm{H}$ & G & $\mathrm{N}$ & $\mathrm{N}$ & A & $\mathrm{N}$ & $\mathrm{LAv}$ \\
\hline 9 & 15 & 25 & 10 & $\mathrm{~L}$ & $\mathrm{P}$ & - & - & - & A & - \\
\hline 10 & 3.5 & 2 & 0 & $\mathrm{H}$ & G & A & $\mathrm{N}$ & $\mathrm{N}$ & - & $\mathrm{Av}$ \\
\hline 11 & 7 & 2 & 0 & $\mathrm{H}$ & G & $\mathrm{N}$ & $\mathrm{N}$ & $\mathrm{N}$ & $\mathrm{N}$ & $\mathrm{LAv}$ \\
\hline 12 & 17 & 1 & 0 & $\mathrm{H}$ & G & $\mathrm{N}$ & $\mathrm{N}$ & A & $\mathrm{N}$ & $\begin{array}{c}\text { Borderline } \\
\text { MR }\end{array}$ \\
\hline 13 & 8 & 5 & 0 & $\mathrm{~L}$ & G & $\mathrm{N}$ & $\mathrm{N}$ & $\mathrm{N}$ & - & - \\
\hline 14 & 1.3 & 12 & 3 & L & $\mathrm{P}$ & - & - & $\mathrm{N}$ & A & - \\
\hline 15 & 9.5 & 10 & 0 & $\mathrm{H}$ & $\mathrm{P}$ & $\mathrm{N}$ & $\mathrm{N}$ & A & - & $\mathrm{Av}$ \\
\hline 16 & 10 & 12 & 0 & $\mathrm{~L}$ & $\mathrm{P}$ & $\mathrm{N}$ & A & $\mathrm{N}$ & $\mathrm{N}$ & Mild ID \\
\hline 17 & 13 & 4 & 0 & $\mathrm{H}$ & G & $\mathrm{N}$ & $\mathrm{N}$ & - & - & - \\
\hline 18 & 4 & 8 & 0 & $\mathrm{~L}$ & $\mathrm{P}$ & $\mathrm{N}$ & $\mathrm{N}$ & - & - & $\mathrm{LAv}$ \\
\hline 19 & 12.5 & 21 & 0 & $\mathrm{~L}$ & $\mathrm{P}$ & $\mathrm{N}$ & $\mathrm{N}$ & A & - & Mild ID \\
\hline 20 & 12 & 6 & 0 & $\mathrm{H}$ & $\mathrm{P}$ & $\mathrm{N}$ & $\mathrm{N}$ & A & - & - \\
\hline 21 & 8.3 & 15 & 3 & $\mathrm{~L}$ & $\mathrm{P}$ & A & $\mathrm{N}$ & A & A & $\begin{array}{l}\text { Borderline } \\
\text { ID }\end{array}$ \\
\hline 22 & 3.3 & 16 & 2 & $\mathrm{~L}$ & $\mathrm{P}$ & A & $\mathrm{N}$ & - & A & $\mathrm{LAv}$ \\
\hline 23 & 7 & 35 & 5 & $\mathrm{~L}$ & $\mathrm{P}$ & A & A & A & A & Mild MR \\
\hline
\end{tabular}

VEP: Visual evoked potential; BAEP: Brain-stem evoked potential; EEG: Electroencephalography; MRI: Magnetic resonance imaging; LAv: Low Average; Av: Average; ID: intellectual disability; N: Normal; A: Abnormal; H: High; L: Low; P: Poor; G:Good. 
that might result in CNS injury were excluded. Age, consanguinity, family and past medical history, and physical and laboratory examination results at presentation were collected from medical records. Treatment regimens and dietary compliance of the patients were also recorded.

Clinical and biochemical parameters that show metabolic control, such as growth parameters, liver size, liver function tests, serum transaminases, glucose, lactate, bicarbonate, uric acid, and lipid levels were studied.

Detailed neurological examination was performed in all patients by a pediatric neurologist.

For psychometric evaluation, Stanford-Binet test was used for five patients aged from 2 to 6 . The Wechsler Intelligence Scale for ChildrenRevised (WISC-R) were used for ten patients aged from 6 to 16 years. The results of IQ tests were classified as follows; above 90: average; between 80 and 89: low average; between 70 and 79: borderline; under 69: mild intellectual disability(ID) ${ }^{10}$.

Electroencephalography (EEG) recordings were obtained in 18 patients using the 10-20 International System with 24 channels bipolar montages (Nihon Kohden Inc, Japan). The EEG recording was carried out during awake state, and hyperventilation and photic stimulation were performed.

Visual evoked potentials (VEP): Subjects were placed $30 \mathrm{~cm}$ away from the monitor. The electrodes were placed on to the scalp according to 10-20 International System. Both eyes were tested separately and the opposite eye was occluded during the test. Checkerboard pattern of black and white squares changing every 50 $\mathrm{ms}$ on the monitor were used as stimuli. The amplitudes and latencies of the P100 wave were evaluated in 21 patients and 21 controls.

Brainstem auditory evoked potentials (BAEP): The test was performed in an acoustically isolated room. The electrodes were placed on to the scalp according to 10-20 International System. The click stimulus with rarefied polarity was monaurally presented at $75 \mathrm{dBHL}$ in a speed of 10 stimuli per second, totaling 1024 stimuli per trace. The values of absolute interpeak I-V latencies were analyzed in 20 patients and 20 controls.
Magnetic resonance imaging (MRI): Fifteen patients had brain MRI (Magnetom Siemens Inc, Germany) including sagittal $\mathrm{T} 1$ and transverse T1 weighted imaging (WI) (TR/ TE; 550/15 ms), transverse and coronal T2 WI (TR/TE; 4000/99 ms), diffusion weighted imaging (DWI) (obtained at three gradient directions with a b maximum of $1000 \mathrm{~s} / \mathrm{mm}^{2}$; TR/TE; 2800/78 ms) and apparent diffusion coefficient (ADC) maps calculated following the data acquisition.

Patients were divided into two groups as those with good and poor metabolic control according to the criteria defined by European Study on Glycogen Storage Disease Type $1^{6}$.

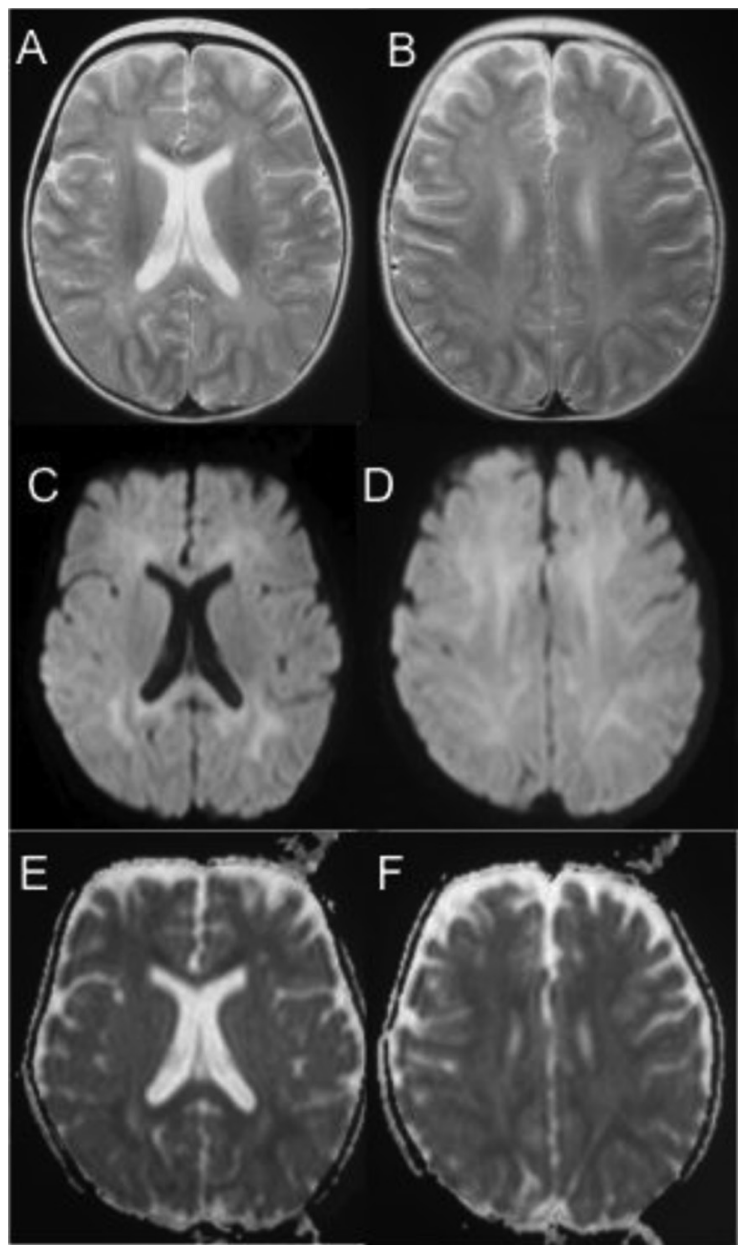

Fig. 1. Cranial MRI of a patient showing axial T2WI (A, B), diffusion WI (C, D) and corresponding apparent diffusion coefficient (ADC) maps (E, F). Diffuse T2 hyperintensity in the periventricular and deep white matter (A, B) including the corpus callosum is seen with diffusion restriction as revealed by hyperintensity on diffusion WI $(\mathrm{C}, \mathrm{D})$ and low ADC values (E, F). 
The patients were assigned as good metabolic control if blood glucose was $>72 \mathrm{mg} / \mathrm{dl}$, triglycerides $<531 \mathrm{mg} / \mathrm{dl}$, uric acid $<6 \mathrm{mg} /$ $\mathrm{dl}$, and lactate $<22.5 \mathrm{mg} / \mathrm{dl}$. Additionally, number of hypoglycemic attacks (assigned as good if less than 5), and dietary compliance were also considered. Equal number of age and sex matched healthy subjects were taken as the control group for evoked potentials evaluation. The results of the evaluations were compared between groups and correlated with parameters of metabolic control.

Statistical Analyses Analyses were performed using the SPSS statistical package (v. 16.0). Data were expressed as numbers (n), frequencies $(\%)$, and means with standard deviations. Categorical variables between groups were analyzed using the $\chi^{2}$ test. Continuous variables were compared by Mann-Whitney $U$ and Kruskal Wallis tests. Correlation analyses were performed with the Spearman test. Differences were considered significant at $\mathrm{p}<0.05$.

\section{Results}

Twenty-three patients with GSD type 1a (18 males and 5 females, age from 1.3 to 17 years, mean $9.1 \pm 4.1$ years), and age and sex matched healthy controls were enrolled in the study. The patients were on follow-up for 0.25-15 year (mean $6.8 \pm 4.2$ years) period. Sixteen patients had parental consanguinity. All of the patients had experienced documented hypoglycemic attacks and eight patients had seizures due to hypoglycemia. Two patients had documented hypoglycemic convulsions in the neonatal period. All of the patients were on frequent meals and uncooked cornstarch dietary treatment. Twelve patients had poor dietary compliance. There were 12 patients in poor metabolic control group and 11 patients in good metabolic control group. The patient characteristics and CNS evaluation results were summarized in Table I.

Neurological examination was normal in all patients (except presence of intellectual disability) and controls. The results of psychometric tests, electrophysiological and radiologic findings of patients and subgroups were summarized in Table II.

Psychometric tests were applied for both verbal and performance abilities. The verbal ability tests revealed $(90.1 \pm 12.1 \mathrm{~min} / \max 76-111)$ three patients with borderline intellectual disability (ID) and three patients with low average IQ. The performance ability tests revealed $(88.4 \pm 18.4 \mathrm{~min} / \mathrm{max} 57-119)$ two patients with mild, three borderline ID, and two patients with low average IQ. The overall test results (verbal and performance ability tests) showed five patients had ID (three mild and two borderline) and four of these patients were in poor metabolic control group.

Electroencephalography revealed slow and irregular background activity for age in six patients, inter-hemispheric asymmetry in five patients and epileptiform focus in one patient who required administration of antiepileptic drugs. Among 10 patients with EEG abnormalities, six were in poor metabolic control group.

Visual evoked potential abnormalities were detected in $7 / 21$ (33.3\%) patients. In particular, five patients had bilaterally, one had right sided, and one had left sided delayed latency

Table II. Summary of the Evaluation Results of the Patients with Glycogen Storage Disease Type 1a and Subgroups with Respect to Metabolic Control

\begin{tabular}{lccc}
\hline CNS evaluations & $\begin{array}{c}\text { Poor metabolic } \\
\text { control }\end{array}$ & $\begin{array}{c}\text { Good metabolic } \\
\text { control }\end{array}$ & All patients \\
\hline Mental retardation & $4 / 8$ & $1 / 7$ & $5 / 15$ \\
EEG abnormalities & $6 / 8$ & $4 / 10$ & $10 / 18$ \\
MRI abnormalities & $5 / 8$ & $0 / 7$ & $5 / 15$ \\
Delayed VEP P100 latency & $4 / 10$ & $3 / 11$ & $7 / 21$ \\
Delayed BAEP I-V interpeak & $2 / 10$ & $0 / 10$ & $2 / 20$ \\
latency & & & \\
\hline
\end{tabular}

VEP: Visual evoked potential; BAEP: Brain-stem evoked potential; EEG: Electroencephalography; MRI: Magnetic resonance imaging. 
of P100 (Fig. 2). Significant difference was observed between the patient and the control groups $(p<0.001)$ but not between the good and poor metabolic control groups $(p>0.05)$. Four of seven patients with VEP abnormalities were in poor metabolic control group.

Brain stem auditory evoked potentials were abnormal in $2 / 20(10 \%)$ patients. Two patients had prolonged I-V interpeak latency and both were in poor metabolic control group (Fig. 3). Significant difference was observed between the patient and the control groups $(p<0.001)$ but not between the good and poor metabolic control groups $(p>0.05)$.

Abnormal findings were detected on brain MRI of five patients (33\%). All patients had bilateral mild white matter T2 hyperintensity. Diffuse periventricular and frontoparietal and occipital deep white matter was affected most

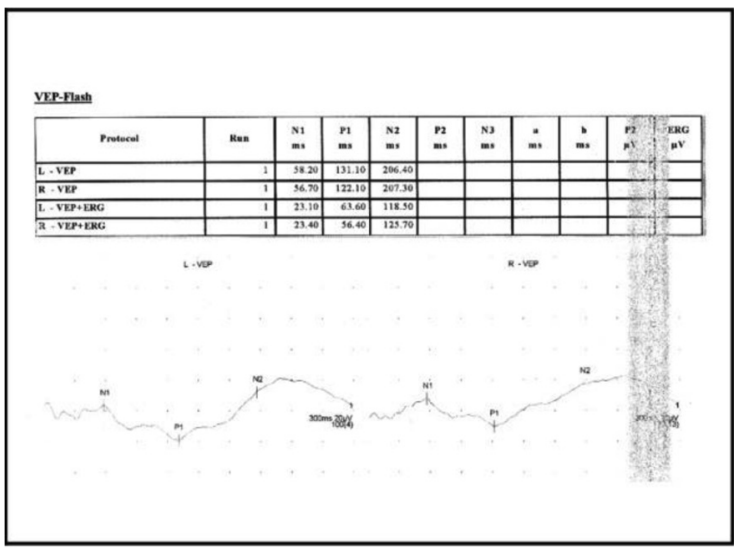

Fig. 2. Visual evoked potential of the patient showing delayed P100 latency

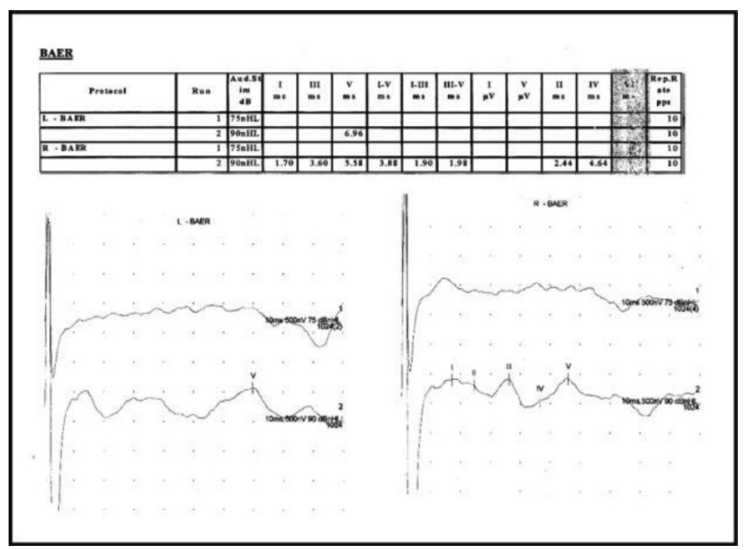

Fig. 3. Brain stem auditory evoked potential of the patient showing prolonged I-V interpeak latency frequently (n:4) and corpus callosum was additionally involved in one patient (Fig. 1 A, B). Diffusion weighted imaging showed restricted diffusion as revealed by low apparentdiffusion co-efficient maps in the patient with corpus callosum involvement (Fig. $1 \mathrm{C}-\mathrm{F}$ ). Two patients had basal ganglia affected, millimetric cysts in the caudate nuclei in one and diffuse high signal intensity of the bilateral basal ganglia on both T1 and T2 WI in other. The patients with MRI abnormalities had frequent hypoglycemic attacks and seizures and all were in poor metabolic control group. Two patients with hypoglycemic convulsions in the newborn period who have also experienced hypoglycemic attacks during childhood had diffuse periventricular, frontoparietal and occipital deep white matter involvement on MRI.

A significant correlation was detected between the number of hypoglycemic attacks and MRI abnormalities. There were no correlations between the number of hypoglycemic attacks and presence of VEP, BAEP, and EEG abnormalities.

\section{Discussion}

Glucose metabolism provides the fuel for physiological brain function through the generation of ATP, the foundation for neuronal cellular maintenance, as well as the generation of neurotransmitters ${ }^{11}$. Patients with GSD type 1a have tolerance to hypoglycemia even at levels of blood sugar at which seizures would be expected, which could be due to utilization of lactic acid or ketone bodies by the brain and hypoinsulinemia secondary to chronic hypoglycemia ${ }^{1}$. There is only one study that evaluates CNS involvement in patients with GSD type 1. Melis et al. ${ }^{12}$ reported neurological examination, psychometric tests, standard electroencephalogram, somatosensory, visual and brain-stem auditory evoked potentials and brain MRI results of nineteen patients with GSD type 1 consistent with variable degree of brain damage. In the present study, we report a systematic evaluation of CNS findings of a larger cohort of GSD type 1a patients on longterm follow up.

The incidence of ID is not as high as expected among GSD type 1a patients. Mental development was low in $3 \%$ and borderline 
in $18 \%$ in a European cohort of GSD type 1a patients ${ }^{6}$. Talente et al. ${ }^{13}$ evaluated educational background of adult GSD type 1 a patients and reported only one patient with special education need. In our cohort, we detected ID in $33 \%$ of the patients, which is higher than the literature reports. These findings can be attributed to high number of patients with poor metabolic control in our cohort.

Pramming et al. ${ }^{14}$ found that EEG was normal when the blood glucose concentration is above $54 \mathrm{mg} / \mathrm{dl}$. At a median blood glucose concentration of $36 \mathrm{mg} / \mathrm{dl}$, the alpha activity $(8-12 \mathrm{~Hz})$ decreased while the theta activity $(4-8 \mathrm{~Hz})$ increased, reflecting a cortical dysfunction. Melis et al. ${ }^{12}$ found occipital spikes in two patients, symmetric parietotemporal slow and sharp waves in two patients and generalized spike waves activity in one patient. We observed EEG abnormalities with unclear clinical significance such as irregular and slow background activity, inter-hemispheric asymmetry in nine patients, which may reflect mild cortical dysfunction. There was only one patient with epileptiform activity on EEG and required treatment for seizures.

Evoked potentials measure the electrophysiological responses of the nervous system to a variety of stimuli. Visual evoked potential measures the conduction of the visual pathways from the optic nerve, optic chiasm, and optic radiations to the occipital cortex. Sannita et al. ${ }^{15}$ found correlation between serum glucose concentration and P100 latencies in normoglycemic volunteers. Melis et al. ${ }^{12}$ found VEP abnormalities in 5/13 (38.4\%) patients with GSD type 1 ; our patients showed similar results seen in $7 / 21$ patients (33\%).

Brain stem evoked potential measures the function of the auditory nerve and auditory pathways in the brain stem. Koh et al. ${ }^{16}$ found abnormal BAEP results in patients with blood glucose level under $45 \mathrm{mg} / \mathrm{dl}$. Melis et al. ${ }^{12}$ found abnormal BAEP results in 3/19 (15.7\%) patients with GSD type 1 . We found increased I-V interpeak latencies in (2/20) $10 \%$ of patients and both were in poor metabolic control group.

Imaging features of patients who suffered from hypoglycemia show various patterns. Barkowich et al. ${ }^{17}$ have reported diffuse cortical and subcortical white matter damage at the parietal and occipital lobes in five hypoglycemic newborns. Murakimi et al. ${ }^{18}$ have reported that neonatal hypoglycemia may cause delayed or abnormal myelination in the parieto-occipital, periventricular and deep white matter, and may cause cerebral cortical atrophy especially in the occipital lobe. However, Burns et al ${ }^{19}$ showed that white matter injury was not only confined to the posterior regions in symptomatic neonatal hypoglycemia but also include; hemorrhage, middle cerebral artery infarction, basal ganglia/thalamic abnormalities, and cortical involvement. Although, only two of our patients had documented hypoglycemia in the newborn period, neonatal type hypoglycemic findings were detected in all patients with abnormal MRI. These findings can be attributed to possible undocumented hypoglycemic attacks during neonatal period in rest of the patients. Melis et al. ${ }^{12}$ found MRI abnormalities characterized by dilation of occipital horns and/ or hyperintensity of subcortical white matter in the occipital lobes in eight of 14 patients with GSD type 1 . In the present study, we observed widespread bilateral and symmetrical white matter involvement and basal ganglia lesions. Previous reports have demonstrated MRI findings in severe hypoglycemia including reversible and irreversible hyperintensity on fluid-attenuated inversion recovery and diffusion restriction on diffusion-weighted imaging in gray matter (cerebral cortex, hippocampus, and basal ganglia) and white matter (posterior limb of the internal capsule, corona radiate, and centrum semiovale) structures in adults ${ }^{20-22}$. In addition to classic MRI findings seen in neonatal hypoglycemia, our findings are also compatible with adult type lesions seen following hypoglycemia. The frequent hypoglycemic attacks in our patients beyond the neonatal period, particularly in patients with poor metabolic control may be responsible for adult type hypoglycemic MRI findings.

Our study has some limitations. Although this is one of the largest cohort studies evaluating CNS in GSD type 1a, low patient number with CNS involvement remains as a limitation for statistical analysis. Additionally, poor patient compliance in young children was a challenge during VEP, BAEP and EEG studies. In five patients under 5 years we performed repeated measurements to overcome this limitation. 
Evaluations including psychometric tests, EEG, VEP, BAEP and MRI demonstrated presence and patterns of CNS involvement in patients with GSD type la even with normal neurologic examination. Recurrent hypoglycemic episodes in neonatal period, infancy and childhood seem to play an important role in the pathogenesis of CNS involvement in GSD type 1a. Accumulation of abnormal results in poor metabolic control group suggests the importance of metabolic control in long-term outcome of these patients.

\section{REFERENCES}

1. Chen YT. Glycogen storage diseases. In: Scriver CR, Beaudet AL, Sly WS, Vale D (eds). The Metabolic and Molecular Basis of Inherited Disease (8th ed). New York: McGraw-Hill; 2001: 1521-1551.

2. Cori GT, Cori CF. Glucose-6-phosphatase of the liver in glycogen storage disease. J Biol Chem 1952; 199: 661-667.

3. van Schaftingen E, Gerin I. The glucose-6-phosphatase system. Biochem J 2002; 362: 513-532.

4. Wolfsdorf JI, Weinstein DA. Glycogen storage diseases. Rev Endocr Metab Disord 2003; 4: 95-102.

5. Froissart R, Piraud M, Boudjemline A M et al. Glucose6-phosphatase deficiency. Orphanet J Rare Dis 2011; 6: 27.

6. Rake JP, Visser G, Labrune P, et al. Guidelines for management of glycogen storage disease type I European Study on Glycogen Storage Disease Type I (ESGSD I). Eur J Pediatr 2002; 161: S112-S119.

7. Weinstein DA, Wolfsdorf JI: Effect of continuous glucose therapy with uncooked cornstarch on the long-term clinical course of type 1a glycogen storage disease. Eur J Pediatr 2002; 161: S35-S39.

8. Rake JP, Visser G, Labrune P, et al. Glycogen storage disease type I: diagnosis, management, clinical course and outcome. Results of the European Study on Glycogen Storage Disease Type I (ESGSD I) Eur J Pediatr 2002; 161: S20-S34.

9. Narisawa $\mathrm{K}$, Igarashi $\mathrm{Y}$, Otomo $\mathrm{H}$, et al. A new variant of glycogen storage disease type I probably due to a defect in the glucose-6-phosphate transport system. Biochem Biophys Res Commun 1978; 83: 1360-1364.
10. Wechsler D. Manual for the Wechsler Intelligence Scale for Children. New York, Psychological Corporation, 1974.

11. Mergenthaler P, Lindauer U, Dienel GA, et al. Sugar for the brain: the role of glucose in physiological and pathological brain function. Trends Neurosci 2013; 36: 587-597.

12. Melis D, Parenti G, Della Casa R, et al. Brain damage in glycogen storage disease type I. J Pediatr 2004; 144: 637-642.

13. Talente GM, Coleman RA, Alter C, et al. Glycogen storage disease in adults. Ann Intern Med 1994; 120: 218-226.

14. Pramming S, Thorsteinsson B, Stigsby B, et al. Glycaemic threshold for changes in electroencephalograms during hypoglycaemia in patients with insulin dependent diabetes. Br Med J 1988; 296: 665-667.

15. Sannita WG, Fatone M, Garbarino S, et al: Effects of physiological changes of serum glucose on the patternVEP of healthy volunteers. Physiol Behav 1995; 58: 1021-1026.

16. Koh TH, Aynsley-Green A, Tarbit M, et al. Neural dysfunction during hypoglycaemia. Arch Dis Child 1988; 63: 1353-1358.

17. Barkovich AJ, Ali FA, Rowley HA, et al. Imaging patterns of neonatal hypoglycemia. Am J Neuroradiol 1998; 19: 523-528.

18. Murakimi Y, Yamashita Y, Matsuishi T, et al. Cranial MRI of neurologically impaired children suffered from neonatal hypoglycaemia. Pediatr Radiol 1999; 29: 2327.

19. Burns CM, Rutherford MA, Boardman JP, et al. Patterns of cerebral injury and neurodevelopmental outcomes after symptomatic neonatal hypoglycemia. Pediatrics 2008; 122: 65-74

20. Aoki T, Sato T, Hasegawa K, et al. Reversible hyperintensity lesion on diffusion weighted MRI in hypoglycemic coma. Neurology 2004; 63: 392-393.

21. Kim JH, Roh JH, Koh SB. Reversible injury of internal capsule and splenium in a patient with transient hypoglycemic hemiparesis. Cerebrovasc Dis 2006; 22: 282-283.

22. Lo L, Tan AC, Umapathi T, et al. Diffusion weighted MR imaging in early diagnosis and prognosis of hypoglycemia. AJNR Am J Neuroradiol 2006; 27 : 1222-1224. 